\title{
Impact of COVID-19: Considerations for individuals with developmental disabilities across major life domains
}

\author{
Kathy Sheppard-Jones ${ }^{\mathrm{a}, *}$, Lauren Avellone ${ }^{\mathrm{b}}$, Phillip Rumrill ${ }^{\mathrm{a}}$ and Hannah Seward ${ }^{\mathrm{b}}$ \\ ${ }^{a}$ University of Kentucky, Lexington, KY, USA \\ ${ }^{\mathrm{b}}$ Virginia Commonwealth University, Richmond, VA, USA
}

Received 1 April 2020

Accepted 13 August 2020

\begin{abstract}
.
BACKGROUND: The impact of COVID-19 and other health pandemics disproportionately affects individuals with developmental disabilities (DD) across a multitude of life domains including health, work, and education.

OBJECTIVE: The purpose of this article is to review some of the factors contributing to the unique impact of pandemics on individuals with DD. Not only are individuals with DD at a heightened risk of contracting a pandemic illness but are also more likely to receive inequitable care and thus the potential to experience more fatal outcomes if contracted. Challenges in receiving equitable services in times of a global crisis are observed in business and educational settings, as well. Many individuals with DD require individualized supports to meet learning and vocational needs. If the quality and continuity of those services are disrupted, individuals with DD risk termination from jobs or slowed progress in meeting educational goals. CONCLUSION: Strategies for addressing concerns related to the impact of a pandemic on different life domains for individuals with DD are discussed.
\end{abstract}

Keywords: Developmental disabilities, COVID-19, vocational rehabilitation, education

\section{Introduction}

In mid-March of 2020, the World Health Organization declared a pandemic in response to the Coronavirus-2019 (COVID-19) outbreak. Early data from countries affected by the virus, such as China and Italy, enabled populations at high risk for fatal outcomes to be identified, including those with underlying health conditions such as cardiovascular disease, lung disease, immunocompromised illnesses, renal disease, liver disease, diabetes, and pregnancy (Chow et al., 2020). Another underlying condition associated with higher risk for more negative outcomes during COVID-19 is neurological/

*Address for correspondence: Kathy Sheppard-Jones, Ph.D., C.R.C., Human Development Institute, University of Kentucky, Lexington, Kentucky, USA. E-mail: kjone@uky.edu. neurodevelopmental disabilities (Chow et al., 2020), an umbrella term for many disorders such as intellectual disability, autism spectrum disorder, genetic syndromes, cerebral palsy, and epilepsy. Roughly one in six children have a developmental disability ([DD]; Zablotsky et al., 2019), and therefore represent a large section of the population left vulnerable during a pandemic. Early trend analyses also find that individuals with DD experience higher risk of acquiring COVID-19 at younger ages (Turk et al., 2020).

The Developmental Disabilities Assistance and Bill of Rights Act (DD Act) of 2000 (Public Law 106-402) defines DDs as conditions which include at least one mental and/or physical impairment, beginning before age 22 , and resulting in substantial functional limitations in three or more major life areas. Impairments to major life activities include those limiting self-care, learning, mobility, communication, 
self-direction, independent living and economic independence (DD Act, 2000). Consequently, individuals with DD often require lifelong supports and services to achieve and maintain daily success in school, work, and community life. Therefore, it is important to consider how the implications of COVID-19 and future pandemics are different for people with DD than they are for other people.

There are several prominent ways in which individuals with DD are adversely affected in times of a global health crisis to an extent larger than that experienced by other populations. COVID-19 elucidated important concerns about the susceptibility of individuals with DD for contracting pandemic viruses at higher rates than other members of the population and trepidations about the manner and fairness in which individuals with DD would be treated if they became ill (Mello, Persad, \& White, 2020). It also raised important questions about how service providers can and should effectively prepare for continuation of services across major life domains, such as health, work and school, in the event of a "shelter in place" order. It is essential for a vast array of stakeholders, including legislators, health care workers, employers and educators, to understand how individuals with DD are at risk of being disproportionately affected during a pandemic in terms of contracting an illness and receiving equitable healthcare, employment support, and educational services.

The purpose of this article is to describe the disparate impact of COVID-19 and other pandemic related illnesses on the population of individuals with DD across several major life domains including healthcare, work, and education. Addressing healthcare first, individuals with DD are at a heightened risk of contracting a pandemic illness and also more likely to experience a severe outcome if they are affected. A review of contributing factors is presented, including a greater reliance on others to assist with health and safety skills, an increased presence of underlying at-risk health conditions, and challenges with an existing healthcare system that is unfamiliar with the unique learning and communication needs of individuals with DD - one that has fallen short in eliminating inequitable service delivery (Bagenstos, 2020). Then, a review of the ways in which a pandemic can disproportionately affect employment outcomes for individuals with DD is presented, touching on factors such as the over-representation of individuals with DD in entry level positions that are eliminated more quickly during economic stress and a lack of access to much needed on-site employment supports and services to install modifications and accommodations aligned with pandemic related protocol changes. Finally, the challenges in transitioning educational services from in-classroom to online that meet the specialized learning needs of individuals with DD are discussed. These include a lack of empirically-based evidence to ensure quality services in the absence of the ability to use established hands-on evidenced based practices (e.g., prompting, modeling, visual cues, etc.). Recommended strategies for a variety of stakeholders are provided.

\section{Heightened risk factors for more negative outcomes for individuals with DD}

There is no doubt that COVID-19 irrevocably changed the world. Daily life has undergone tremendous shifts since its onset, and the entire globe was suddenly forced to grapple with how to live with new norms. While necessary orders to flatten the curve came from medical professionals and politicians in the form of directives to monitor and report symptoms, increase hand washing and sanitation routines, and adhere to social distancing practices (Centers for Disease Control and Prevention [CDC], 2020), the disability community faced real fears and challenges in meeting those recommendations. Recognizing that individuals with DD are at a heightened risk of contracting viruses such as COVID-19 is imperative, and the reasons for this imperative are discussed below.

\subsection{Assistance with activities of daily living}

Many individuals with DD require assistance completing activities of daily living (Hilgenkamp, van Wijck, \& Evenhuis, 2011; MacDonald, Ross, McIntyre, \& Tepfer, 2017), which exacerbates risks for contracting the virus. A person with DD may rely on family or paid caregivers for assistance. In fact, more than $70 \%$ of individuals with an intellectual/developmental disability live with a family caregiver (Braddock et al., 2013). Caregivers often help individuals with DD complete tasks, and in the mere act of doing so may impede both party's ability to follow social distancing guidelines. For example, assisting an individual with getting dressed or brushing teeth would violate the six feet apart physical distancing guideline as a matter of necessity. This places individuals with DD at an increased need to be in closer proximity to others who may be carriers of a pandemic virus. 
Those with DD who have the capacity for completing such tasks on their own are often accustomed to completing these activities as part of structured routines. Therefore, although the individual may be able to practice safe hygiene, she or he may not independently increase the autonomy of safe habits to the elevated frequencies recommended during a health crisis (e.g., increased hand-washing) or adapt to new suggestions (e.g., refrain from touching facial regions or wear a mask) in the appropriate contexts. Individuals with DD may therefore be less likely to perform these skills independently outside of their typical routines, and with enough diligence to ensure that they are sufficiently protecting themselves from infection. For these reasons, it is imperative that, first and foremost, society recognize that individuals with DD already have a higher risk of contracting a serious illness.

\subsection{Communication differences}

If an individual with DD does contract a pandemic virus, differences in communication can also negatively impact care. The receptive and expressive language abilities of individuals with DD often differ from those without disabilities (MacDonald et al., 2017). If the person is feeling ill, he or she may not have the language to articulate symptoms to others or to understand standard screening procedures implemented by healthcare workers enough to respond accurately. This presents two concerns. The first is a failure to seek treatment when needed and the second is the consequences of inadequate treatment that can subsequently be the result of communication differences.

Both of these concerns place individuals with DD at a greater risk of improper care, inadequate care or lack of care, all of which can have dire consequences during a global health crisis. Communication differences can lead to inequities in healthcare treatment if professionals are unable to identify strategies for addressing these barriers. The Americans with Disabilities Act (ADA) of 1990 requires that state and local governments, along with businesses and nonprofit agencies that serve the public, communicate effectively with people who display differences in expressive and receptive language - the goal being to ensure that communication with a person with a disability is as effective as communication with those without disabilities (ADA, 1990). However, not all entities serving the public are trained in how to alter language, use plain language or to use different communication platforms in order to meet the needs of a person with different language abilities. This can jeopardize the quality of care received when the dangers for health and safety are highest. Hospital restrictions on visitors have led to unavailability of support persons knowledgeable in the needs of the individual to aid in the provision of quality health care in hospital settings. As part of the resolution to a case filed in Connecticut, an Executive Order will ensure access to needed support staff so that a person's rights are not compromised (HHS.gov, 2020).

\subsection{Secondary conditions as risk factors}

In general, a high prevalence of co-morbid health conditions is observed among individuals with DD (Must et al., 2014). A myriad of factors contribute to this phenomenon, including financial barriers to adequate healthcare, difficulties navigating the complexities of the medical system (Ervin, Hennen, Merrick, \& Morad, 2014), sedentary lifestyles, unhealthy diets, medication side effects (Must et al., 2014), and a lack of transportation to and from medical appointments. Individuals with DD are less likely than individuals without disabilities to attend routine check-ups (Zworth, Selick, Durbin, Casson, \& Lunsky, 2019) and are therefore more likely to receive a diagnosis after the comorbid-condition has become more advanced and (therefore) more detrimental to their health. As a result, many individuals with DD are likely to develop secondary conditions that compound their risk of experiencing more negative outcomes of COVID-19 (Chow et al., 2020).

\subsection{Health systems are unprepared to deal with the needs of people with DD}

Health systems have made some strides in attempting to meet the needs of those with DD by promoting interdisciplinary healthcare services and educating healthcare providers on how to more effectively serve individuals with DD (Ervin et al., 2014). However, in the wake of a pandemic that consumes resources beyond what is routinely expected, there is the potential for this progress to break down. This heightened risk for systemic failure comes with serious implications for patients who have DD. Individuals with DD may be unable or less likely to see their usual medical providers who are more familiar with their communicative abilities and health histories on account of travel and physical engagement restrictions installed during a pandemic. Also, 
interdisciplinary team members who are not medical professionals and are required to work remotely, such as advocates or liaisons, can become less integrated at the point of care.

As healthcare providers become overwhelmed with meeting the needs of the general population during a public health crisis like COVID-19, the resource of time can become scarce. Opportunity to read resources on the healthcare needs of those with DD becomes less likely. The ability of healthcare providers to communicate effectively with people with DD is paramount, as people with DD may have substantial difficulty making health decisions without guidance and support (Ervin et al., 2014). Moreover, as telehealth services replace in-person site visits to reduce risk of exposure, individuals with DD and others who are unfamiliar with, lack access to, and are uncomfortable with these technology platforms are more likely to be excluded from necessary care.

\subsection{Potential for discrimination that denies medical treatment}

Although there has been considerable discussion of at-risk groups during COVID-19 with respect to age (older), gender (male), and underlying conditions (Chow et al., 2020), there has been less information concerning how other demographics such as race and disability status are affected. Since it is well documented that individuals with disabilities have a pervasive history of discrimination (ADA, 1990; IDEA, 2004), now is an ideal time to recognize this point and ensure that we move forward in a way that provides equity and high regard for individuals with DD during global crises.

For example, complaints have recently been filed in several states regarding Standards of Care in the Coronavirus crisis. As a result of numerous welljustified complaints from disability rights advocates, the director of the United States Office of Civil Rights (OCR) has already been compelled to issue guidance to make sure that states and providers maintain healthcare ethics and humanity. In Alabama, the early crisis standards of care (now rescinded) indicated that hospitals should not consider people with severe or profound intellectual disabilities as candidates for mechanical ventilator support (Bagenstos, 2020). Utah recommended exclusions on transfer of care for individuals with more profound cognitive limitations. In Tennessee, those with neuromuscular diseases, such as spinal muscular atrophy, who would be expected to need help with activities of daily living were projected to be excluded from receiving care in the event of an equipment shortage (Mello, Persad, \& Douglas, 2020). Similar discriminatory practices toward individuals with disabilities were observed in protocols in other states across the U.S. including New York, Pennsylvania, Massachusetts, Kansas and Washington (Bagenstos, 2020; Mello et al., 2020).

The OCR guidance reminds us that nondiscriminatory laws such as ADA (1990) still apply, regardless of a shift in circumstances. Equitable treatment in health care is always critical and always a right, but now inequitable care on account of discrimination has the potential to result in higher stakes and irreversible negative outcomes for individuals with DD, including loss of life. Several knee-jerk responses in healthcare, such as those described above, underscore the legitimacy of the disability community's concerns regarding potential discrimination. Further, unfair practices could markedly affect other major life areas for individuals with DD who also have a history of discrimination in work, school and housing, all of which have the potential to re-emerge during events such as pandemics.

\section{Risk of disproportionate impact across life domains for individuals with DD}

COVID-19 has brought about numerous changes to the way society conducts business, attends school, and engages in recreation and leisure. Although these adjustments came with challenges for most of the population, the transition came with a distinct set of difficulties for those with DD. The set of supports and services these individuals receive at work and school to promote success was not necessarily designed to be adaptable for alternate contexts, and it has not needed to be until now. Looking toward the future, employment service providers and educators will have to anticipate how to bridge supports across contexts in preparation for future outbreaks. These providers will also have to ensure that individuals with DD are receiving their fair and legal entitlements and not subject to discrimination in light of changing practices. A discussion of each is presented below.

\subsection{Employment}

In the United States, all non-essential personnel whose employment was not terminated, began working remotely from home in March of 2020. Individuals with DD employed in community jobs 
during this time experienced several barriers. First, and unfortunately, most individuals with DD work in entry level positions (Zworth et al., 2019) such as restaurant and retail jobs, and these were some of the first industries to shut their doors during the pandemic. Therefore, individuals with DD as a population were already at a higher likelihood of being out of work and without pay early in the pandemic. This loss of income for basic needs is a grave concern. With few businesses remaining open, the prospect of finding other work in similar professions to generate income is low. Those who are eligible for unemployment face having to complete complex procedures for filing, and many individuals with DD may require assistance in order to complete the paperwork or to even know that they are eligible for unemployment. This makes them less likely to end up finding alternate income during the crisis. A lack of income can negatively affect mental well-being, as well as create or exacerbate underlying at-risk health conditions.

Secondly, individuals with DD who work in entry level positions at businesses which remain open during a pandemic, such as grocery stores or pharmacies, often have employment support staff providing onthe-job training or follow-along support to help with job retention. Depending on the agency for which the employment support staff work, they may be unable, due to pandemic restrictions, to meet with the employee with DD and provide employment support on-site. This is a particularly detrimental time for an employee with DD to be without support as new rules regarding social distancing, direction of movement down aisles, changes to return policies, and requirements to wear gloves and masks are likely to be in place that an employee with DD may need assistance to learn and implement with fidelity. Failure to do so can have somber effects, such as job termination or the experience of harmful health outcomes.

Lastly, individuals with DD who work in higher skilled positions would likely be required to begin working remotely, which often requires additional skills such as learning to use teleconferencing software, remote log-in requirements, new procedures for documenting work tasks and other novel skills they may need accommodations or modifications to perform. Such accommodations and modifications are usually assessed and installed with the help of a trained employment specialist (Chen, Sung, \& Pi, 2015), who, again, may be suddenly limited in what can be provided. Until now, preparation for how to successfully support individuals with DD in the workplace during a pandemic has not been explored, but there are important concerns to consider in terms of the impact on safety and job retention. While employers and businesses start to feel the economic impact of COVID-19 or other pandemics, they will be forced to make difficult decisions about whom to keep on the payroll just like health professionals are forced to make difficult decisions about whom to treat. This makes it especially important that the population of individuals with DD is not unequally targeted, as individuals with disabilities already experience alarmingly higher rates of unemployment compared to individuals without disabilities when there is no pandemic (U.S. Bureau of Labor Statistics, 2020)

\subsection{Education}

Federal regulations require that students who meet eligibility criteria for a disability receive specialized educational services (Individuals with Disabilities Education Act, 2004). These include the receipt of individualized learning objectives and evidencedbased practices for teaching different skills. The transition of materials for students without disabilities focuses more heavily on independent work and reduced direct instruction time from teachers themselves. This format does not meet the more hands-on instructional needs of students with disabilities. Students with DD may have different learning needs (Yakkundi, Dillenburger, Goodman, \& Dounavi, 2017) met by different modes of teaching that include strategies such as modeling steps in an activity, using prompting to complete tasks, installing visual supports in a classroom environment as cues, or delivering tangible reinforcement for correct answers. These tools are, of course, much more difficult for a teacher to implement remotely or through a teleconferencing app and therefore, students with DD may experience slower progress meeting educational goals.

The CDC released a guidance document to help K12 schools plan, prepare, and respond to COVID-19 (National Center for Immunization and Respiratory Diseases [NIRD], 2020). These guidelines suggest the development of proactive strategies that prevent the disruption of educational services to the maximum extent possible. These strategies include ensuring that all students (including those with DD) have access to technology, modifying curriculum to eLearning platforms, developing feasible ways to incorporate adult supervision and collaboration during tele-instruction sessions, and developing ways to trouble shoot technology failure while tech support 
is more limited and busier than usual (NIRD, 2020). To date, there is no significant evidence base identifying best practices for teaching students with DD remotely. Research that does exist focuses primarily on reducing behavioral problems through telehealth formats (Lindgren et al., 2016) rather than educational programming. This leaves students with DD at higher risk of falling behind in education than their peers without disabilities.

A pandemic does not change the fact that children with disabilities in public schools are legally and ethically entitled to services that meet criteria for a free and appropriate public education (FAPE) and the standards set forth by the Rehabilitation Act of 1973 and the Individuals with Disabilities Education Act (IDEA, 2004). And while teachers do their best to smooth the transition to online teaching, there is much left for educators and researchers to examine regarding the most efficacious way to provide high quality services in a new modality for students with DD. This conundrum is not just limited to K-12 populations. Colleges and universities serving students with disabilities will also have to find new ways to offer parallel accommodations and modifications as courses transfer from on-campus to online-only formats. Although it is certainly a struggle for all students to adjust to an entirely different mechanism for learning in environments beyond the classroom, stakeholders must make sure that the unique learning needs of students with DD are not lost and simply lumped into the universal solutions implemented as a response to a pandemic.

In summary, the prospect of mistreatment in health, employment and education, whether intentional or unintentional, during times of a health crisis is a possibility that needs attention. Spreading awareness of the likelihood for inequitable conduct and highlighting the vulnerability of individuals with DD during a pandemic is the first step toward prevention. The second step is inciting action on the part of stakeholders large and small. This requires collaborative work from legislators and direct service providers.

\section{Reporting violations}

The stage is set. What does this mean in light of the coronavirus, and more specifically COVID-19 in 2020 and beyond for people with developmental disabilities? On March $28^{\text {th }}, 2020$, the OCR at the US Department of Health and Human Services provided a reminder to everyone that, during this pandemic, all of the protections provided under laws like the ADA, the Affordable Care Act and Section 504 of the Rehabilitation Act still apply. These reminders are an attempt at helping to ease concerns that have been rising in the disability community, and in response to some states' actions that could be interpreted as out of sync with the aforementioned laws (Bagenstos, 2020; Mello et al., 2020). Violations should be actively reported in a timely manner to help ensure that people with disabilities do not have to worry about being denied basic services. To that end, OCR is requesting that complaints be submitted to their office at hhs.gov/OCR.

\section{Strategies to support the rights of people with developmental disabilities}

As individuals with DD face substantially more disrupted lives and increased risks of discrimination in essential life areas as a result of the pandemic, it is germane to discuss effective tactics to addressing these concerns. These tactics can be used to minimize disparate effects on people with DD. Ensuring that individuals with DD continue a high standard of care during a global crisis begins with stakeholders understanding their distinct needs and ends with actions to meet those needs. Table 1 provides a list of example support strategies that should be employed to combat potential injustice.

\subsection{Future directions}

The impact of a pandemic affects all aspects of daily life. Employment, school and organized community activities not only serve to advance knowledge and self-sufficiency but also provide a social outlet for spending time with others and working toward common goals. For many individuals with DD, these outlets serve as their primary avenue for socialization. In the absence of such activities in their daily routines, there is a real risk for some to feel very lonely or disconnected. The ability to connect with others using socially distant platforms will vary as some individuals with DD are fluent with social media, texting, and video apps. Still others may not own or have access to the costly technology that is needed to use those social platforms. Or, others with DD may not know how to independently operate them even if they could access the technology. From a mental health standpoint, it is certainly important that individuals with DD are able to combat loneliness, 
Table 1

Support strategies for individuals with DD in different settings during a pandemic

\begin{tabular}{|c|c|}
\hline Stakeholders in life domains & Support strategies \\
\hline Medical professionals & $\begin{array}{l}\text { - Treatment should not be denied based upon presence of a disability. } \\
\text { - Do not underestimate or overestimate an individual with DD's ability } \\
\text { to understand medical conversations. } \\
\text { - Ask for permission from the individual with DD before discussing information } \\
\text { about them with others. } \\
\text { - Confirm if the individual with DD has a trusted caregiver, guardian, advocate, liaison } \\
\text { or translator to include in medical conversations. } \\
\text { - Do not assume the individual with DD already knows changes in routine or procedures as a result } \\
\text { of a pandemic and be sure to explain any changes at the beginning of a visit. } \\
\text { - Use plain language and non-medical jargon. } \\
\text { - Provide accommodations that enable continued care for individuals with DD such as allowing } \\
\text { a trusted caregiver to join into a hospital visit when restrictions on number of people } \\
\text { in a clinical setting are restricted. } \\
\text { - Ask caregivers about picture cards, icons, dry erase boards, or other assistive technologies that } \\
\text { an individual might need to bring with them to a medical visit to use for communication. } \\
\text { - Encourage health literacy by making health messaging distributed to the public accessible } \\
\text { to the entire population including people with DD by modifying language and presentation } \\
\text { in ways that accounts for disabilities. } \\
\text { - Educate colleagues and other medical professionals so everyone is acutely aware they people } \\
\text { with disabilities have equal rights to COVID-19 testing and treatment. } \\
\text { - Provide accommodations that will enable individuals with DD to be less afraid } \\
\text { and more comfortable seeking treatment. } \\
\text { - Include individuals with disabilities on ethics teams and discussion panels to ensure their } \\
\text { interests are represented during times of crisis. } \\
\text { - Become proficient in the Core Competencies on Disability for Health Care Education } \\
\text { available at https://www.ahce.org }\end{array}$ \\
\hline $\begin{array}{l}\text { Employers and } \\
\text { business } \\
\text { administrators }\end{array}$ & $\begin{array}{l}\text { - Be generally aware of what type of support an employee with DD needs to learn and } \\
\text { perform various tasks so similar supports can transition with the individual during times } \\
\text { of crisis. } \\
\text { - Consult with an employment specialist early in a pandemic about different types of schedules, } \\
\text { technology, visual cues, or other forms of support could help the individual learn changes } \\
\text { in protocol or procedures as a result of a pandemic. } \\
\text { - Proactively ask what remote employees with DD might need to alter their work such as simplified } \\
\text { directions for remote log in or how to access work databases. } \\
\text { - Allow extra training for an individual with DD to learn and adjust to abrupt } \\
\text { changes in employment protocol } \\
\text { - Offer simplified versions of pandemic instructions and procedures that may help a person } \\
\text { with DD learn new routines such as wearing a mask or maintaining distance from others at work. } \\
\text { - Ask what accommodations an employee with DD might need to minimize exposure to a virus, } \\
\text { including a temporary shift change or restructuring of job activities during the length of the pandemic. } \\
\text { - Encourage an open door policy which allows an employee with DD to feel comfortable } \\
\text { asking questions and expressing concerns regarding changes afflicted by the pandemic. }\end{array}$ \\
\hline Educators & $\begin{array}{l}\text { - Establish a plan for continuity of educational services that meet disability specific needs. } \\
\text { - Proactively plan for technology failure that would disrupt educational services. } \\
\text { - Prepare materials to be sent home on short notice (e.g., communication icons, picture schedules, etc.) } \\
\text { - Develop student specific informational resources about individual students with DD so staff } \\
\text { can quickly implement programs in the event of staff absenteeism during a pandemic } \\
\text { when staff sick days are likely higher. } \\
\text { - Consider the provision of necessary services such as the distribution of meals to students } \\
\text { in need during times of crisis } \\
\text { - Develop a communication system with parents to evaluate the effectiveness } \\
\text { of at-home programing strategies } \\
\text { - Enact data driven processes to scientifically track and evaluate student progress outside } \\
\text { of only IEP goals. } \\
\text { - Establish online resource banks with colleagues to serve as a hub for sharing helpful resources } \\
\text { as quickly as possible. }\end{array}$ \\
\hline
\end{tabular}

anxiety and depression during times of isolation, but it is also essential that they are able to connect with others in the event of emergencies. More than ever, providing novel ways to offer social connections and making technology accessible to individuals with DD are critical. 
There is also much to do in the way of research. Knowing how to install better evidenced-based supports and services for individuals with DD in a variety of life domains that preserve quality of care for medical services and ensure equitable treatment in education and employment is key to preventing disproportionately unfavorable outcomes. There is also much to do in the way of education. Education can help eradicate the need for making one's own best guess when it comes to decisions about who should receive medical treatment, retain employment or receive proper educational services. As mentioned earlier, despite one in six individuals having DD (Zablotsky et al., 2019), the American Medical Association still has no training criterion on DD for physicians at all levels of training. Core competencies on disability for health care education have been created to provide guidance on baseline professional knowledge when providing care to patients who have disabilities (Alliance for Disability in Health Care Education, 2019). However, there remains a need to better understand DD in medical education and for employers to have better directions and support in how to equitably treat employees with disabilities when circumstances change.

Disability organizations are coming together at the state and national levels along with self-advocates and family members to help ensure that disability discrimination does not occur during a global health situation. There is a valuable opportunity to learn from COVID-19. There is true anxiety for people with disabilities and for families of people with disabilities. Multiple systems are being tested to the extreme. There is so much health and economic uncertainty in all aspects of life that access to good information, using flexibility and accommodations as much as possible, and working in concert with each other will help the community innovate, learn and grow regarding how to respond effectively and fairly during a pandemic toward individuals with DD.

\section{Conflict of interest}

None to report.

\section{References}

Alliance for Disability in Health Care Education. (2019). Core competencies on disability for health care education. Peapack, NJ: Alliance for Disability in Health Care Education. https://www.ahce.org
Americans with Disabilities Act of 1990, 42 U.S.C. $\$ 12101$ et sea. (1990). https://www.ada.gov/pubs/adastatute08.htm

Bagenstos, S. R. (2020, May 15). May hospitals withhold ventilators from COVID-19 patients with pre-existing disabilities? Notes on the law and ethics of disabilitybased medical rationing. SSRN. https://papers.ssrn.com/sol3/ papers.cfm?abstract_id=3559926

Braddock, D., Hemp, R., Rizzolo, M. C., Tanis, E. S., Haffer, L., Lulinski, A., \& Wu, J. (2013). State of the states in developmental disabilities, 2013: The great recession and its aftermath. American Association on Intellectual and Developmental Disabilities.

CDC COVID-19 Response Team. (2020, April 3). Preliminary estimates of the prevalence of selected underlying health conditions among patients with coronavirus disease 2019-United States, February 12-March 28, 2020. Morbidity and Mortality Weekly Report, 69(13), 382.

Centers for Disease Control and Prevention, Division of Viral Diseases. (2020, April 24). How to protect yourself \& others. Author. https://www.cdc.gov/coronavirus/2019-ncov/preventgetting-sick/prevention.html

Chen, J. L., Sung, C., \& Pi, S. (2015). Vocational rehabilitation service patterns and outcomes for individuals with autism of different ages. Journal of Autism and Developmental Disorders, 45(9), 3015-3029. https://doi.org/10.1007/s10803015-2465-y

Developmental Disabilities Assistance and Bill of Rights Act of 2000, Pub. L. No. 106-402, 114 Stat. 1677 (2000). https://acl. gov/sites/default/files/about-acl/2016-12/dd_act_2000.pdf

Ervin, D. A., Hennen, B., Merrick, J., \& Morad, M. (2014). Healthcare for persons with intellectual and developmental disability in the community. Frontiers in Public Health, 2, 83. https://doi.org/10.3389/fpubh.2014.00083

Health and Human Services. (2020). OCR resolves complaints after state of Connecticut and private hospital safeguard the rights of persons with disabilities to have reasonable access to support persons in hospital settings during COVID-19. https://www.hhs.gov/about/news/2020/06/09/ocrresolves-complaints-after-state-connecticut-private-hospitalsafeguard-rights-persons.html

Hilgenkamp, T. I., van Wijck, R., \& Evenhuis, H. M. (2011). (Instrumental) activities of daily living in older adults with intellectual disabilities. Research in Developmental Disabilities, 32(5), 1977-1987. https://doi.org/10.1016/j.ridd. 2011.04.003

Individuals with Disabilities Education Act of 2004, 20 U.S.C. $\$ 1400$ et seq. (2004). https://uscode.house.gov/view. xhtml?path=/prelim@ @itle20/chapter33\&edition=prelim

Lindgren, S., Wacker, D., Suess, A., Schieltz, K., Pelzel, K., Kopelman, T., Lee, J., Romani, P., \& Waldron, D. (2016). Telehealth and autism: Treating challenging behavior at lower cost. Pediatrics, 137(Supplement 2), S167-S175. https://doi. org/10.1542/peds.2015-2851O

MacDonald, M., Ross, S., McIntyre, L. L., \& Tepfer, A. (2017). Relations of early motor skills on Age and socialization, communication, and daily living in young children with developmental disabilities. Adapted Physical Activity Quarterly, 34(2), 179-194. https://doi.org/10.1123/apaq.2015-0091

Mello, M. M., Persad, G., \& White, D. B. (2020). Respecting disability rights - Toward improved crisis standards of care. The New England Journal of Medicine, 383(5), e26. https://doi. org/10.1056/NEJMp2011997 
Must, A., Curtin, C., Hubbard, K., Sikich, L., Bedford, J., \& Bandini, L. (2014). Obesity prevention for children with developmental disabilities. Current Obesity Reports, 3(2), 156-170. https://doi.org/10.1007/s13679-014-0098-7

National Center for Immunization and Respiratory Diseases (NCIRD), Division of Viral Diseases. (2020, March 19). Interim guidance for administrators of US K-12 schools and child care programs: Plan, prepare, and respond to Coronavirus Disease 2019 (COVID-19). https://www.cdc.gov/coro navirus/2019-ncov/community/schools-childcare/guidancefor-schools.html\#role

Turk, M.A., Landes, S.D., Formica, M.K., \& Goss, K.D. (2020). Intellectual and developmental disability and COVID-19 case fatality trends: TriNetX analysis. Disability and Health Journal. https://doi.org/10.1016/j.dhjo.2020.100942

U.S. Bureau of Labor Statistics. (2020, February 26). Persons with a disability: Labor force characteristics summary.
United States Department of Labor. https://www.bls. gov/news.release/disabl.nr0.htm

Yakkundi, A., Dillenburger, K., Goodman, L., \& Dounavi, K. (2017, September). User Centered Reading Intervention for Individuals with Autism and Intellectual Disability. In AAATE Conf. (pp. 249-256)

Zablotsky, B., Black, L. I., Maenner, M. J., Schieve, L. A., Danielson, M. L., Bitsko, R. H., Blumberg, S. J., Kogan, M. D., Boyle, C. A. (2019). Prevalence and trends of developmental disabilities among children in the US: 2009-2017. Pediatrics, 144(4), e20190811. https://doi.org/10.1542/peds.2019-0811

Zworth, M., Selick, A., Durbin, J., Casson, I., Lunsky, Y. (2019). Improving care for adults with intellectual and developmental disabilities: Role of clerical staff. Canadian Family Physician, 65(Supplement 1), S8-S10. 